\title{
POSITION DETERMINATION OF GRAVITATIONAL WAVE SOURCES WITH LISA
}

\author{
M. Peterseim ${ }^{1}$, O. Jennrich ${ }^{1}$, B.F. Schutz ${ }^{2}$, and K. Danzmann ${ }^{1,3}$
}

${ }^{1}$ Institut für Atom- und Molekülphysik, Universität Hannover, Appelstraße 2, 30167 Hannover, Germany

${ }^{2}$ University of Wales, College of Cardiff, United Kingdom

${ }^{3}$ MPI für Quantenoptik, Hans-Kopfermann-Str. 1, 85748 Garching, Germany

\section{ABSTRACT}

LISA is a spaceborne laser interferometer for the detection and observation of gravitational waves, currently under study by ESA. A brief introduction of the main features of this detector, concentrating on its oneyear orbital motion around the Sun is given. The amplitude as well as the phase of a gravitational wave is modulated due to that motion, allowing us to extract information from the signal. The detection of monochromatic gravitational waves based on the well-known signal detection theory is simulated, focusing on estimating the angular parameters of the source. The results of the semi-analytic calculations give the angular resolution of LISA. (C) 2003 Published by Elsevier Ltd on behalf of COSPAR.

\section{INTRODUCTION}

The LISA mission consists of six spacecraft in a plane inclined $60^{\circ}$ with respect to the ecliptic and along a circle with a radius of $3 \times 10^{9} \mathrm{~m}$ (Danzmann et al., 1996). Two spacecraft each will be placed at three points on this circle, forming an equilateral triangle with a baseline of $5 \times 10^{9} \mathrm{~m}$ which rotates clockwise (viewed from the Sun) around its center, with a period of one year. The whole constellation describes an approximately circular orbit at a distance of $R=1 \mathrm{AU}$ from the Sun and following the Earth in its orbit by $20^{\circ}$.

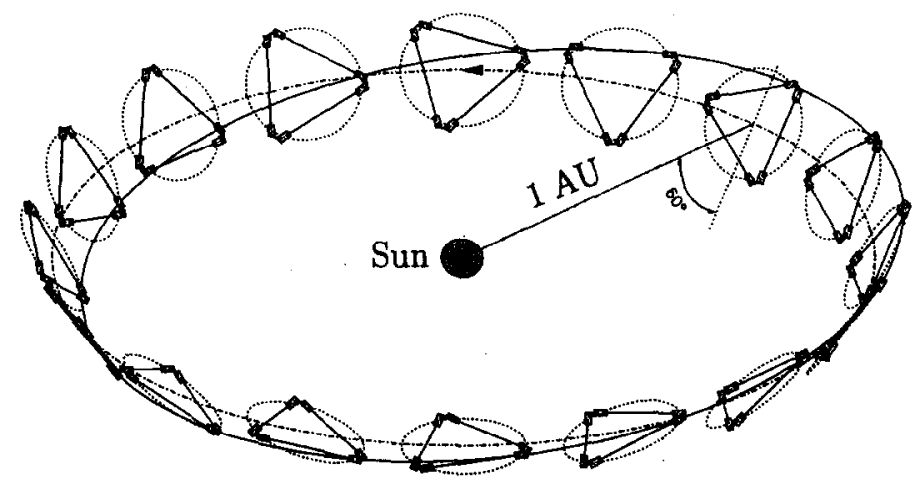

Fig. 1. Annual revolution of the LISA configuration around the Sun, describing a cone with a half opening angle of $60^{\circ}$

Four such spacecraft will form a Michelson-type interferometer, here of course with $60^{\circ}$ between the arms. The two close spacecraft at a corner will have separate lasers that are phase-locked so as to represent the "beamsplitter" of a Michelson. The distances are measured from the test masses housed freely floating in the six drag-free spacecraft. The distant spacecraft are equipped with lasers of their own, phase-locked to 
the incoming laser beam, so they represent the (active) end-mirrors of the interferometer. It will be shown that a single non-moving detector reveals no information about the parameters that characterize the source of the gravitational wave. But the described motion of LISA leads to an amplitude modulation of the signal (Peterseim et al., 1996). All the information about the source parameters is then contained in the variation of the gravitational wave signal or the detector response to that signal, respectively.

\section{SIGNAL AND RESPONSE}

Gravitational waves of measurable strength are emitted only when large cosmic masses undergo strong accelerations, for instance in the orbits of a (close) binary star. The effect of such a gravitational wave is an apparent strain in space, transverse to the direction of propagation, that makes distances between test bodies shrink and expand by small amounts. That strain in space can be expressed mathematically by adding a time-dependent part to the space-time metric:

$$
\begin{aligned}
g_{\mu \nu} & =\eta_{\mu \nu}+h_{\mu \nu} \\
& =\left(\begin{array}{cccc}
-1 & 0 & 0 & 0 \\
0 & 1 & 0 & 0 \\
0 & 0 & 1 & 0 \\
0 & 0 & 0 & 1
\end{array}\right)+\left(\begin{array}{llll}
h_{00} & h_{10} & h_{20} & h_{30} \\
h_{01} & h_{11} & h_{21} & h_{31} \\
h_{02} & h_{12} & h_{22} & h_{32} \\
h_{03} & h_{13} & h_{23} & h_{33}
\end{array}\right)
\end{aligned}
$$

An interferometer of the Michelson type is an ideal instrument to measure the strain caused by the wave. When the wave passes trough the apparatus the light beams in the two arms undergo different phase shifts, leading to a net phase difference at the output port. This phase difference, usually denoted as the detector response, is a simple linear combination of the elements of $h_{\mu \nu}$ for gravitational waves that are well within the LISA detection bandwidth (Peterseim et al., 1996):

$$
\delta \Phi=\frac{2 L \pi}{4 \lambda} \sqrt{3}\left[\left(h_{22}-h_{11}\right) \sqrt{3}+h_{12}+h_{21}\right]
$$

where $L$ is the armlength and $\lambda$ is the laser wavelength. In the early stage of the above mentioned binary systems the gravitational wave signal can be assumed to be purely monochromatic. Therefore, we confine our paper to the class of monochromatic waves. If LISA was at rest relative to the source, the effect of such a wave impinging on the interferometer would be a periodic vari-

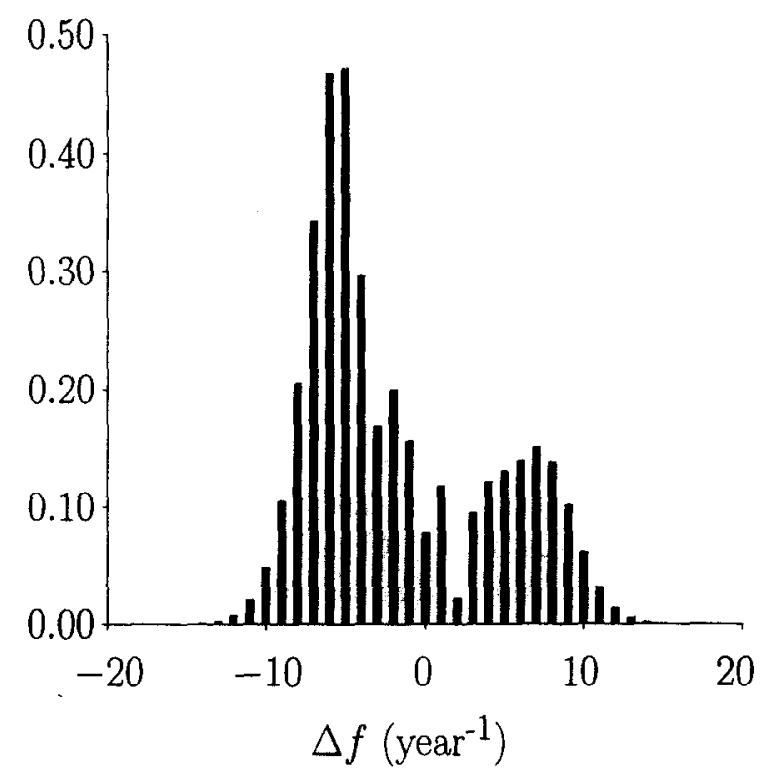

Fig. 2. Detector response to a gravitational wave at $3 \mathrm{mHz}$. The source location is $(\theta, \phi)=\left(\frac{\pi}{4}, 0\right)$. ation of the phase difference. The Fourier transform of this response is a constant peak in the corresponding frequency bin. This kind of response, apart from the amplitude height, would be the same for all sources in the same frequency bin revealing not much information. The above mentioned motion of the detector leads to phase and amplitude modulation of the response, from which more information can be inferred.

Firstly, the translatory motion of the detector relative to the source is Doppler shifting the signal. This leads to a time-dependent phase shift of the response that is harmonic with a period of one year. Secondly, the amplitude of the measured phase difference depends on the source location relative to the interferometer, i.e. the detector response pattern is not isotropic but has a kind of "peanut shape" (Saulson, 1994). When LISA 

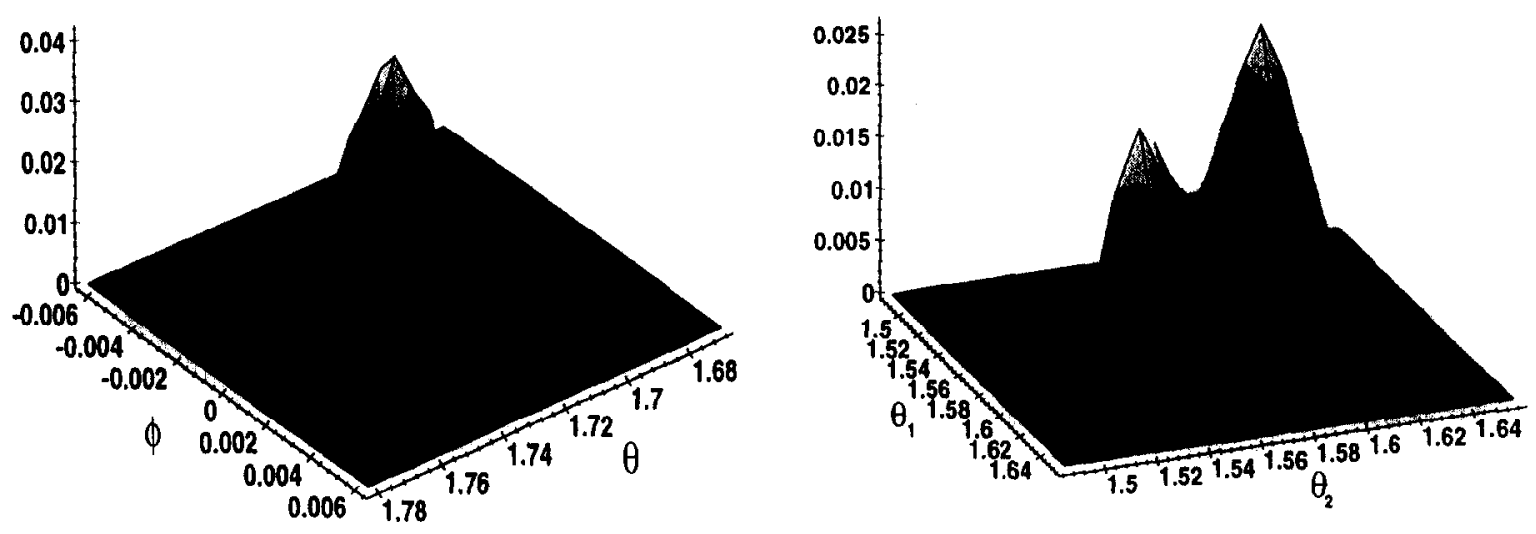

Fig. 3. Probability distribution $\mathcal{P}\left(s, \vec{\mu}_{t}\right) \mathrm{d}^{M} \mu$ for the parametrizations $(\theta=0.55 \pi, \phi=0)$ (left) and $\left(\theta_{1}-\right.$ $0.488 \pi, \theta_{2}=0.512 \pi$ ) (right). The simulation is carried out for a cross-polarized wave at $3 \mathrm{mHz}$ and a signal-to-noise ratio of 115 .

rotates around the Sun this pattern is rotated and the detector's response to a signal of fixed amplitude becomes harmonic in time with frequencies from 1 year $^{-1}$ to 4 year $^{-1}$. Together these modulations will spread a sharply peaked monochromatic signal into a set of sidebands separated from the carrier at integer multiples of the fundamental frequency 1 year $^{-1}$ (Figure 2). This sideband structure is characteristic for the source, and by comparing it with templates it is possible to estimate all the parameters that are needed to characterize the source. The following section gives the mathematics of the comparison technique.

\section{PARAMETER ESTIMATION THEORY}

A gravitational wave signal or the corresponding detector response $\Delta \Phi$ is characterized by an $M$-dimensional set of parameters $\mu_{1}, \ldots, \mu_{M}$, denoted as $\vec{\mu}_{0}$. Some of these parameters may be e.g. the position of the source in the sky $\left(\mu_{1}=\theta, \mu_{2}=\phi\right)$. The measured data stream $s$ is a time series containing the response $\Delta \Phi$ plus additional noise $n$ that is assumed to be white stationary Gaussian noise within the detector's bandwidth. The noise amplitude is determined by the LISA noise spectrum $\mathcal{S}_{n}$ (Danzmann et al., 1996).

The first step in parameter estimation is to construct a probability density function (PDF) $\mathcal{P}$. A measured noisy response $s\left(\vec{\mu}_{0}\right)$ is characterized by the real parametrization $\vec{\mu}_{0} . \quad \mathcal{P}$ is a function of a trial set of parameters $\vec{\mu}_{t}$, in such a way that $\mathcal{P}\left(s, \vec{\mu}_{t}\right) \mathrm{d}^{M} \mu$ is the probability for $\vec{\mu}_{t}$ to fulfill the condition $\left|\vec{\mu}_{t}-\vec{\mu}_{0}\right|<$ $\mathrm{d}^{M} \mu$, which means for $\vec{\mu}_{t}$ to be located inside an $M$-dimensional sphere of radius $\mathrm{d}^{M} \mu$ centered at $\vec{\mu}_{0}$. Figure 3 shows as an example a PDF for a 2-dimensional $\vec{\mu}_{0}$. Without noise in $s$, the PDF would of course be a product of delta-like functions. Detailed information about $\mathcal{P}$ can be found in (Cutler and Flanagan, 1994) and (Peterseim et al., 1997). The second step is to evaluate the PDF : One has to find a data-processing algorithm in order to estimate the measured values of the parameters; one is the so-called Bayes estimator, a kind of mean value generalized to the PDF:

$$
\hat{\vec{\mu}}_{\text {Bayes }}=\int \vec{\mu}_{t} \mathcal{P}\left(\vec{\mu}_{t}, s\right) \mathrm{d}^{M} \mu
$$

Due to the additional noise in the data stream the estimated values will be slightly faulty. In Bayes' theory of parameter estimation the error in measurement is taken to be the width of the probability density function 
$\mathcal{P}(\vec{\mu}, s)$ for the measured value $\hat{\vec{\mu}}$, i.e. the variance-covariance matrix (Cutler and Flanagan, 1994):

$$
\begin{aligned}
\Sigma_{i j} & =\int\left(\mu_{i}-\hat{\mu}_{i}\right)\left(\mu_{j}-\hat{\mu}_{j}\right) \mathcal{P}(\vec{\mu}, s) \mathrm{d}^{M} \mu \\
\delta \mu_{i} & =\sqrt{\Sigma_{i i}}
\end{aligned}
$$

\section{ANGULAR RESOLUTION}

Angular resolution means the accuracy of the position determination of gravitational wave sources, assuming all other parameters are perfectly known. That means $\vec{\mu}_{0}$ is 2-dimensional and consists only of the two Euler angles $\theta$ and $\phi$ (Figure 3). The figure of merit to express the angular resolution is the canonical combination for weakly correlated angles, the solid angle of uncertainty $\mathrm{d} \Omega=\sin \theta \delta \theta \delta \phi$. For the detection of a $3 \mathrm{mHz}$ monochromatic linearly polarized gravitational wave and a signal-to noise ratio of 100 , the value for the solid angle falls in the range of $1 \ldots 16 \mu$ sterad, depending on the position of the source in the sky (Figure 4). The Euler angles are defined in a reference frame with its axis $\theta=\pi / 2$ located in the ecliptic and its axis $\phi=0$ pointing toward LISA at the start of data taking.

One major assumption of the previous section is that there is only one source falling within each frequency bin. Due to the large number of sources in the low-

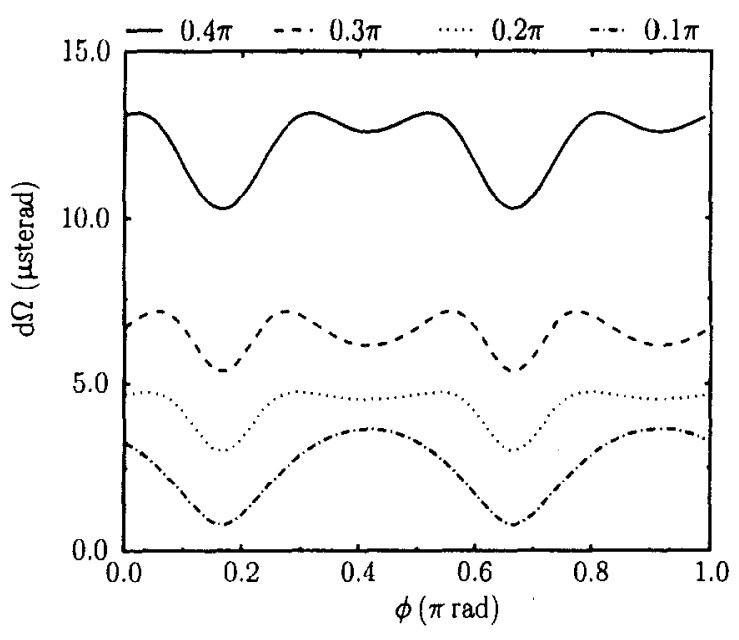

Fig. 4. Error of position as function of $\phi$ for several values of $\theta$. Calculated a plus-polarized $3 \mathrm{mHz}$ wave with a signal-to-noise ratio of $\rho=$ 115.

source of much lower amplitude is present in the same bin, it can be regarded as additional noise. In the case of two sources with comparable amplitude contained in one frequency bin, the parametrization $\vec{\mu}$ has to be doubled in dimension. An example of the PDF for the simultaneous detection of two identical (frequency, polarization, and amplitude) sources very closely localed can be found in Figure 3 (right). The dimension of $\vec{\mu}$ is again two, but now the entries are the two $\theta$ angles of the two templates. The PDF has two maxima, as the two sources are interchangeable.

\section{REFERENCES}

Cutler C. and E.E. Flanagan, Gravitational Waves from Merging Compact Binaries: How accurately can one extract the binary's parameters from the inspiral waveform?,Phys. Rev. D, 49, pp. 2658-2697 (1994).

Danzmann K. and the LISA Study Team, LISA Pre-Phase A Report, MPQ 209 (1996).

Finn L.S., Detection, Measurement and Gravitational Radiation, Phys. Rev. D, 46, pp. 5236-5249 (1992).

Peterseim M., O. Jennrich, and K. Danzmann, Accuracy of Parameter Estimation of Gravitational Waves with LISA, Proceedings of the Symposium on Fundamental Physics in Space, Class. Quantum Grav., 13, pp. A279-A284 (1996).

Peterseim M., O. Jennrich, B.F. Schutz, and K. Danzmann, Angular resolution of LISA, Proceedings of the $1^{\text {st }}$ International LISA Symposium, Class. Quantum Grav., to be published (1997).

Saulson P.R., Fundamentals of Interferometric Gravitational Wave Detection, World Scientific Publishing, Singapore, pp. 9-27 (1994).

Thorne K.S., Gravitational Radiation, in 300 Years of Gravitation, ed. S.W. Hawking and W. Israel, Cambridge University Press, Cambridge, pp. 330-458 (1989). 